\title{
Implementation and Evaluation of Consumer-Oriented Lifelog Service Using Daily Receipts
}

\author{
Seiki Tokunaga, Shinsuke Matsumoto, Masahide Nakamura \\ Graduate School of System Informatics, Kobe University \\ 1-1, Rokkodai-cho, Nada, Kobe, Hyogo 657-8501, Japan \\ tokunaga@ws.cs.kobe-u.ac.jp, \{shinsuke, masa-n\}@cs.kobe-u.ac.jp
}

\begin{abstract}
In this paper, we design and implement a consumer-oriented service platform called ReceiptLogService to store and use their receipt log by consumers. ReceiptLogService assumes receipt data as a lifelog. The receipt lifelog may provide gaining awareness of economic for consumers. We also develop a social network Web application called ReciLog that extensively uses the ReceiptLogService platform. ReciLog extensively supports every end user to review daily life from financial and living aspects. We then conducted an experimental evaluation of the ReciLog with actual subjects. The experimental results showed that it was interesting and useful for end users to review and share the receipt log.
\end{abstract}

\section{Categories and Subject Descriptors}

H.3.5 [Information Storage And Retrieval]: Online Information Services-Web-based services, Data sharing;

\section{General Terms}

Human Factors, Experimentation, Design

\section{Keywords}

Lifelog, receipt, Web services, database, social network service

\section{INTRODUCTION}

The purchase history of consumers have been widely used by many companies to investigate and determine their sales strategy [1]. By analyzing enormous quantity of the purchase history, the companies can grasp useful information such as hot selling items, preference of the consumers, and cross-selling items. Based on the information, the companies can take efficient strategies in optimizing stocks as well as making attractive goods arrangement. Thus, the purchase history has been a key for the companies not only to increase the profits, but to improve consumer satisfactions.

Permission to make digital or hard copies of all or part of this work for personal or classroom use is granted without fee provided that copies are not made or distributed for profit or commercial advantage and that copies bear this notice and the full citation on the first page. To copy otherwise, or republish, to post on servers or to redistribute to lists, requires prior specific permission and/or a fee.

iiWAS2011, 5-7 December, 2011, Ho Chi Minh City, Vietnam.. Copyright 2011 ACM 978-1-4503-0784-0/11/12 ...\$10.00.
On the other hand, we consider that the purchase history should not be limited to the business use only. The history is also useful for the consumers themselves. Users can record the purchase history from his daily receipts, which we call receipt log. By collecting sufficient amount of the receipt log, the user can review his purchase history and may find economic information such as the bedrock price of certain goods. Integrating the receipt log with other data sources can yield more sophisticated information. For example, by using the body metrics (e.g., weight, fat) with the receipt $\log$, the user may review the relationship between his health condition and meals.

Traditionally, the daily receipts have been recorded as the bookkeeping, typically written in papers/notebooks, or managed by stand-alone applications. However, the primary purpose of the bookkeeping is to grasp the daily income and outgo, which covers the financial aspect only. Beyond the conventional bookkeeping, our goal is to extend the receipt $\log$ for many other value-added services, including daily-life improvements, health checking, social networks services, etc.

Wide variety of lifelog services are provided. The main purpose of lifelog is to record every personal history as digital data for gaining awareness of him/herself. The personal history includes diary, photos, health status and so on. Furthermore, a variety of lifelog services provided in the internet such as Twitter and Flickr. Users can easily records and shares his/her lifelog.

The goal of this paper is to provide a consumer-oriented receipt lifelog service. In this paper, we design and implement service platform (called ReceiptLogService) to store and use receipt $\log$ for consumers. ReceiptLogService is a Web service that allows external applications to store and retrieve the receipt log easily. Furthermore, we also implement a social network Web application, called ReciLog, which uses the ReceiptLogService. However the ReceiptLogService provides just preliminary features for application developers, it is not easy for end-users to use the service directly. Using the ReciLog the end-user can review own daily receipts just like blog, and view the summary of the purchase with graphs. We also conduct a usability experiment with actual subjects, in order to evaluate the advantage and limitation of the ReciLog.

\section{PRELIMINARIES}

Many companies have taken the management and sales strategies based on the purchase history of consumers. In modern systems, the purchase history is collected in database by using the Point of Sales (POS) system. The data items 
Table 1: Classification of lifelog services

\begin{tabular}{l|c|cc}
\hline \multicolumn{2}{l}{} & \multicolumn{2}{c}{ Owner } \\
\cline { 3 - 4 } \multicolumn{2}{c|}{} & self & share \\
\hline lifelog & single & single-self & single-share \\
data sources & multiple & multiple-self & multiple-share \\
\hline
\end{tabular}

of the purchase history generally include date and time of purchase, age of the user, location, and so on.

These are methods to use purchase history for business use, so they aren't able to use for consumer themselves. Users need platform to record their receipt by oneself and service which is useful for users by using daily receipt, so that users use their purchase history. Our long-term goal is to make full use of the purchase history to develop various value-added services for consumers themselves.

\subsection{Lifelog}

The lifelog [2] is a social activity to record various human life in terms of events, status, and relationships. The recorded log is used to look back and improve daily life, to discover the identity, to determine actions, etc.

In this paper, we propose a classification method of lifelog services based on two attributes: data source and owner, as shown in Table 1.

The data source can take two values: single or multiple. The single means that the service uses a single data source of lifelog. On the other hand, the multiple represents that the service aggregate multiple sources of lifelog for more sophisticated service. The owner also takes two values: self or share. The self means that the user uses the own lifelog data for himself. Whereas, the share means that the lifelog is shared by multiple users as in the social network services.

By combining the two attributes, we presented four classes of lifelog services. For example, a single-self service refers to a service where a user recalls his lifelog only. A multipleshare represents the one that a user publish different types of lifelog among the community.

\subsection{Reserch Goal and Approach}

The goal of this paper is to provide a benefit of recording daily receipts for consumers themselves. Recording receipts may provide gaining awareness of economic for consumers. Moreover consumer may look back his/her health status because most receipts include meal information.

To meet this goal, we design and implement a service platform, called ReceiptLogService, for storing receipt log (section 3). This platform is constructed by Receipt scanner, Receipt $\log D B$ and Receipt $\log A P I$. We also develop a social network Web application called ReciLog that extensively uses the ReceiptLogService platform to ensure usability of ReceiptLogService for end-users (section 4).

\section{ReceiptLogService}

\subsection{Application Overview}

We consider that recording the daily receipts by consumers themselves can be a consumer-oriented lifelog service (singleself) that reflects their purchase history. Also, sharing the receipt log data within the community may achieve a social lifelog service (single-share). Moreover, integrating the receipt $\log$ with other data sources (e.g., pictures, body

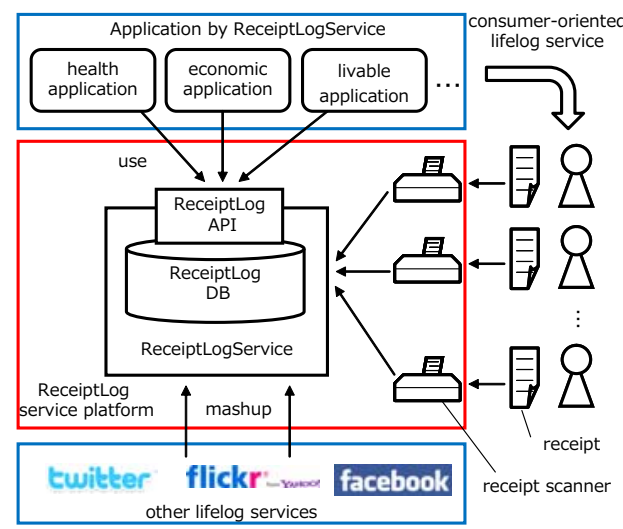

Figure 1: ReceiptLogService architecture

measurements, etc.) may implement sophisticated services (multiple-self or multiple-share).

To facilitate the development of such lifelog services, we design and implement a service platform, called ReceiptLogService, for storing and retrieving data of the daily receipts. Figure 1 shows the architecture of ReceiptLogService. First, each user digitizes daily receipts by using a receipt scanner [3]. Then, data items such as a shop name, purchase date and time, a list of items, are extracted and stored in a in a global database, called ReceiptLogDB. The data can be accessed from external applications via ReceiptLogAPI. The ReceiptLogAPI has been implemented as Web service, so that the receipt log can be accessed from various platforms.

\subsection{Receipt Scanner}

As a receipt scanner, we use a commercial product Yasasiku Kakeibo [3] which includes a receipt scanning device and receipt data management software developed by Media Drive Corporation. First, Yasasiku Kakeibo reads receipt image using a scanning device. Then, receipt data items such as a shop name, purchase date, time, and a list of bought items, are extracted from the receipt image by Optical Character Reader. Each receipt data item are stored to ReceiptLogDB.

\subsection{ReceiptLogDB}

Receipt data items, scanned by the receipt scanner, are stored to ReceiptLogDB. By storing the receipt data in the shared ReceiptLogDB, these data are shared with multiple users. We designed and implemented the ReceiptLogDB by following.

Design schema of ReceiptLogDB: ReceiptLogDB has five tables. Figure 2 depicts an ER diagram of the ReceiptLogDB. A box represents an entity (i.e., table), consisting of an entity name, a primary key and attributes. Database instances are shown at bottom of each box. We enumerate instances below each entity to support understanding. A line represents a relationship between entities, where $+-\epsilon$ denotes a parent-children relationship, and $+\ldots .$. denotes a reference relationship. The five tables are described as follows.

User table: The user table stores personal information for each user. Concretely, the information includes user ID, user name, height, weight and more. 


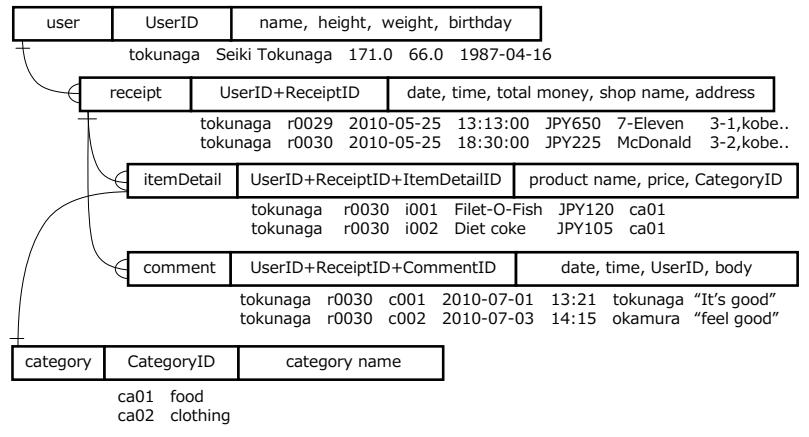

Figure 2: Data schema of ReceiptLogDB

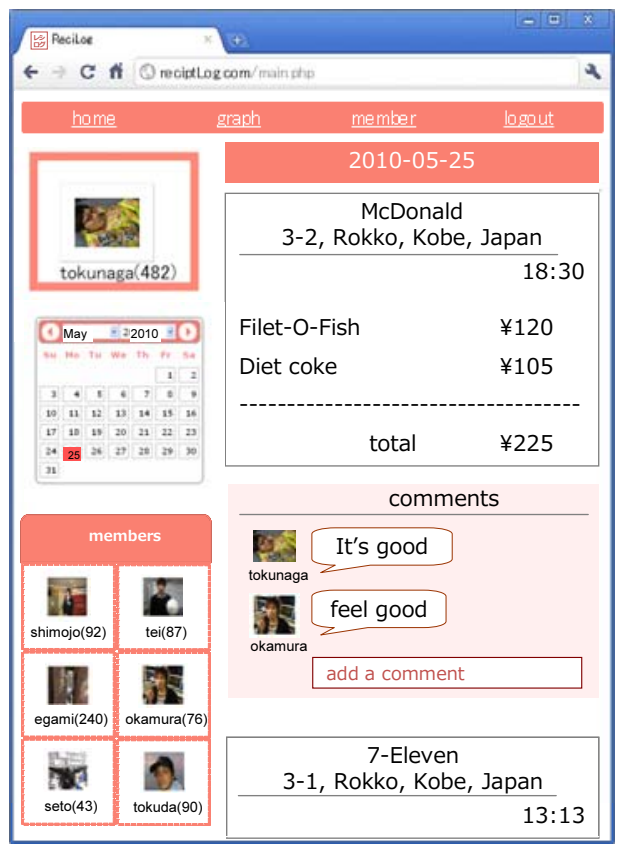

Figure 3: Screenshot of ReciLog: reviewing self receipts

Receipt table: The receipt table stores receipt information for each receipt. One receipt has some breakdowns which are stored in breakdown table as a childrenparent relationship. Basically we assume that a query of ReceiptLogDB includes user name frequently. So we set both ReceiptID and UserID as primary keys for improving access performance.

Breakdown table: Breakdown table stores detailed information of each receipt. As the receipt table, we added both ReceiptID and UserID as primary keys.

Category table: Category table stores product categories such as food, clothing, daily necessities and so on.

Comment table: This table stores user comments made by other users. Users can make comments to each receipt with each other like a social network service.

\subsection{ReceiptLogAPI}

ReceiptLogAPI allows access to the ReceiptLogDB by simple and abstract API invocation for ReceiptLogService users. Examples of ReceiptLogAPIs are follows.

- getReceipt (user, date) returns a list of receipts of a specified user issued on specified date.

- getReceiptDetail(user, ReceiptID) returns details of a specified user and receipt.

The API can be invoked by standard Web service protocols (i.e., SOAP and REST).

\section{ReciLog: SOCIAL NETWORK WEB AP- PLICATION}

\subsection{Application Overview}

Next, we also develop a social network web application, called ReciLog, as a client application of ReceiptLogService. The ReciLog is constructed by the ReceiptLogAPI and has three main features. The main feature is the reviewing feature, which allows the consumers to review daily receipts just like the blog. So we can recall receipt log easily as if we look back on the diary. Figure 3 shows screenshots of the Recilog. The next subsection explains details of the features.

\subsection{Features of ReciLog}

ReceiLog has following three features: reviewing feature, sharing feature and reporting feature. According to our classification presented in Table 1, each feature is classified into single-self, single-share and single-self and single-share, respectively.

\subsubsection{Reviewing Feature}

The reviewing feature helps a user to review the daily receipts easily. The user can add comments to each receipt, to describe the explanation of the goods, the motivation of the purchase, and the impression and so on. Once the login is succeeded from login screen, the blog-like reviewing screen appears (Figure 3 ). In this screen, the user can review the latest receipts and add some comments. By clicking the calendar at the left side of screen, the user can move back to the older receipts issued on the specified date.

\subsubsection{Sharing Feature}

The sharing feature of the ReciLog allows the user to share the receipt log with friends. This feature is analogous to the one of the conventional social network services, where a user can form a community. The lower-left corner of Figure 3 enumerates the friends of the user. By clicking the icon of a friend, the user moves to the receipt log of the friend, where the user can add comments to the purchase of the friend. This yields communication caused by the purchase.

\subsubsection{Reporting Feature}

Finally, the reporting feature presents statistics of the receipt log using graphs and tables.

Using the reporting feature, the user can easily outlook receipts. Moreover, integrating the reporting feature with the sharing feature, we can obtain consumption statistics within the community.

\section{EXPERIMENTAL EVALUATION}


Table 2: Result of Task 1: What did you think to review your receipts by youself ?

\begin{tabular}{r|rllll}
\hline Score & (None) & 1 & 2 & 3 & 4 (Very) \\
\hline Q1: Interesting? & 0 & 1 & 3 & 0 \\
\hline Q2: Useful? & 0 & 1 & 2 & 1 \\
\hline
\end{tabular}

\subsection{Experiment Overview}

In order to evaluate the ReciLog, we have conducted an experiment, where subjects actually use the ReciLog to review their own receipt log. In the experiment, we gave two types of tasks to the subjects. The first task (Task 1) is to evaluate the single-self aspect of the ReciLog, where each subject reviews own receipt log from economic and health aspects. The second task (Task 2) is to see the single-share aspect, where the subjects share the receipt log within the community.

Four master course students participated in the experiment. They were all single male. Two of the four were living with parents, and two were living alone in apartments. The total number of receipts registered in the RecieptLogService was 471 , which had been collected for 12 months. After the tasks were finished, we collected a questionnaire from every subject. The questionnaire consisted of the following questions for each of Tasks 1 and 2.

Q1: Was the task interesting for you? $(1,2,3,4)$

Q2: Did you find it useful to improve your life? $(1,2,3,4)$

Q3: Was the service useful to review your economic aspect?

Q4: Was the service useful to review your health aspect?

The questions Q1 and Q2 were marked by the 4-levels scale. For Q3 and Q4, the subjects answered by comments.

\subsection{Result of Task 1: ReciLog as Single-Self Lifelog Service}

First, we show the result of Task 1 , where each subject reviews own receipt log. Table 2 shows the frequency distribution of the questions Q1 and Q2. The average scores of Q1 and Q2 were 2.75 and 3.00, respectively. This result shows that the subjects felt interesting and found it useful to review the receipt log.

The positive comments includes that "I could grasp the monthly expenses that had been unaware of." Also, a subject said that "I became aware of the lack of vegetables."

On the other hand, a subject commented negatively that "The reporting feature was too poor to conduct the detailed analysis." Another subject said that "I couldn't review diet." The reason is that he did not have receipts for the dinner, since he was living with parents. Thus, the ReciLog can treat single users under the present circumstances. In the future, it will be necessary for the ReciLog to group multiple users as a unit such as family.

We consider there are two reasons why the average score of Q1 and Q2 went no further than 3.00. The first reason is that the amount of receipt log was not sufficient. The second is that the reporting function is too poor to derive interesting results. As for registering the receipt data, we completely rely on the motivation of the subjects, which imposes not a little overhead to the subjects. So, it is necessary to enrich the system so that it can motivate the users to register the receipts spontaneously.
Table 3: Result of Task 2: What did you think to share your receipts with friends?

\begin{tabular}{r|rllll}
\hline Score & (None) & 1 & 2 & 3 & 4 (Very) \\
\hline Q1: Interesting? & 0 & 1 & 2 & 1 \\
\hline Q2: Useful? & 0 & 1 & 3 & 0 \\
\hline
\end{tabular}

\subsection{Result of Task 2: ReciLog as Single-Share Lifelog Service}

Next, we show the result of Task 2, where the subjects share the receipt log within the community. Table 3 shows the frequency distribution of Q1 and Q2. The average score of Q1 and Q2 were 3.00 and 2.75, respectively. From the result, we can see that the subjects were a little bit interested in sharing the receipt log.

Positive comments include that "I was interested in sharing the receipts," since he lives with parents and he can compare his dietary habit with others who are living alone. On the other hand, negative comments include the one that "It is not interesting at all to see others receipts as they are." This is because the ReciLog currently supports to review the others receipts and to add the comments only. In the future, we plan to implement more powerful features that can take full advantages of social network (e.g., trend analysis, sales information).

\section{CONCLUSION}

In this paper, we have developed a consumer-oriented lifelog service by using daily receipts. Concretely, we have design and implemented a service platform called ReceiptLogService which provides basic APIs for recording and reading a receipt log. Next, we also developed a social network Web application, called ReciLog, which allows consumers to review their daily receipts.

We then conducted an experimental evaluation of the ReciLog with actual subjects. The experimental results showed that it was interesting and useful for end users to review and share the receipt log.

Our future work is explained in subsection 5.2 and 5.3. It is also important to conduct the evaluation with more users with various ages, genders, and roles.

\section{ACKNOWLEDGMENTS}

This research was partially supported by the Japan Ministry of Education, Science, Sports, and Culture [Grant-inAid for Scientific Research(B) (No.23300009), Young Scientists (B) (No.21700077), Research Activity Start-up (No.22800042)], and Hyogo Science and Technology Association.

\section{References}

[1] Micael J. A. B. and Gordon S. L. Data Mining Techniques: For Marketing, Sales, and Customer Relationship Management. Wikley Publishing Inc., 1999.

[2] Jim G., Gordon B., and Roger L. Mylifebits: A personal database for everything. Communication of the ACM, 49:88-95, 2006.

[3] MediaDrive Inc. Yasasiku Kakeibo. http: //mediadrive.jp/products/ykakeibo/index.html. 